\title{
Radical Tympanomastoidectomy
}

National Cancer Institute

\section{Source}

National Cancer Institute. Radical Tympanomastoidectomy. NCI Thesaurus. Code C96213.

A therapeutic surgical procedure that involves the removal of the posterior and superior walls of the external auditory canal, the remnants of the tympanic membrane, and the middle ear ossicles (except the stapes footplate), resulting in the conversion of these spaces into a common cavity. 